\title{
CLINICAL RESEARCH ARTICLE Relation of secondhand smoke exposure to vascular phenotypes in children and adolescents
}

\author{
Michelle M. Harbin ${ }^{1}$, Aaron S. Kelly ${ }^{2,3}$, Donald R. Dengel ${ }^{1,3}$, Kyle D. Rudser $^{3,4}$, Nicholas G. Evanoff ${ }^{1,3}$ and Justin R. Ryder ${ }^{2,3}$
}

BACKGROUND: Subclinical cardiovascular risks of secondhand smoke (SHS) exposure among children and adolescents remains insufficiently described.

METHODS: This was a cross-sectional study of 298 children and adolescents $\left(48.0 \%\right.$ male, body mass index: $\left.27.0 \pm 8.9 \mathrm{~kg} / \mathrm{m}^{2}\right)$, including 49 self-reported cases with SHS. Arterial elasticity and stiffness (distensibility, compliance, incremental elastic modulus [IEM]) were obtained via ultrasound imaging in the abdominal aorta, brachial, and carotid arteries. A one-way analysis of variance compared differences between groups, and multiple linear regression adjusted for covariates.

RESULTS: SHS was associated with lower abdominal aorta diameter distensibility (aDD) $(13.4 \pm 3.6 \%$ vs. $16.0 \pm 5.2 \%, p=0.009)$ and abdominal aorta cross-sectional distensibility (aCSD) $(28.8 \pm 8.3 \%$ vs. $35.1 \pm 12.2 \%, p=0.009)$, as well as higher abdominal aorta IEM (alEM) (1241 \pm 794 vs. $935 \pm 388 \mathrm{mmHg}, p=0.001)$. After adjustment for covariates, aDD $(p=0.047), \operatorname{aCSD}(p=0.040)$, and alEM ( $p=0.017)$ remained significant; this significance persisted with the additional adjustment of percent body fat. Measures of brachial and carotid compliance and distensibility were not associated with SHS.

CONCLUSIONS: SHS was associated with abdominal aorta stiffness; the majority of vascular measures within the brachial and carotid artery remained unaffected following adjustment for covariates, including hypertension and adiposity. SHS may predispose individuals to increased abdominal aorta stiffness, an artery previously reported to exhibit increased susceptibility to atherosclerosis.

Pediatric Research (2020) 87:760-766; https://doi.org/10.1038/s41390-019-0627-x

\section{INTRODUCTION}

Involuntary exposure to environmental, secondhand smoke (SHS) increases cardiovascular disease (CVD) risk among adults, ${ }^{1}$ predominately mediated by platelet activation that subsequently causes increased risk of thrombosis formation, ${ }^{2}$ endothelial dysfunction, ${ }^{3-5}$ arterial stiffness, ${ }^{6-8}$ and systemic inflammation. ${ }^{9}$ The growing body of evidence on the adverse effects of SHS among nonsmokers has led to increasing momentum for policies advocating for smoke-free environments. ${ }^{10}$ Population-level data from the National Health and Nutrition Examination Survey (NHANES) reported a decline in the percentage of nonsmokers with serum cotinine levels between 0.05 and $10 \mathrm{ng} / \mathrm{mL}$ from $87.5 \%$ in 1988 to $25.2 \%$ in $2014 .{ }^{11}$ While the restriction of public smoking has substantially decreased SHS exposure, people continue to be exposed, including children. ${ }^{12}$ Between 2013 and 2014, the prevalence of SHS among nonsmokers was significantly higher among children aged 3 to 11 years old as compared to adults older than 20 years old. ${ }^{11}$

Epidemiological studies report that children exposed to SHS are predisposed to having poor mental health, ${ }^{13}$ poor cognitive function, ${ }^{14}$ neurobehavioral disorders, ${ }^{15}$ and metabolic syndrome, $^{16}$ as well as asthma and various respiratory diseases. ${ }^{17}$ Data in adults support the notion that cardiovascular effects of SHS are similar to active smoking among adults, ${ }^{1}$ while the deleterious effect of SHS on cardiovascular health among children and adolescents has been less extensively researched. Children and adolescents exposed to SHS are at increased risk of developing carotid artery atherosclerotic lesions, ${ }^{18}$ as well as increased carotid intima-media thickness and decreased brachial flow-mediated dilation (FMD), ${ }^{19}$ later in adulthood. Another study reported that young children exposed to SHS displayed elevated blood pressure. ${ }^{20}$ The adverse effects of SHS on arterial elasticity, including ultrasound-derived measures of compliance, distensibility, and incremental elastic modulus, are yet to be researched among children and adolescents. The purpose of this analysis was to evaluate differences in measures of vascular structure and function within the carotid, brachial, and abdominal aorta among children and adolescents who had a self-reported history of SHS exposure, as compared to unexposed children and adolescents. We hypothesized that children and adolescents exposed to SHS will exhibit greater levels of many subclinical CVD risk factors.

\section{METHODS}

Two hundred and ninety-eight participants (143 males) between 8 and 18 years old who participated in a cross-sectional study assessing CVD risk among children and adolescents throughout a range of body mass index (BMI) values were included in this retrospective analysis. Participants were recruited from the University of Minnesota Masonic Children's Hospital Pediatric

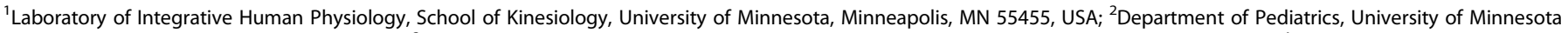

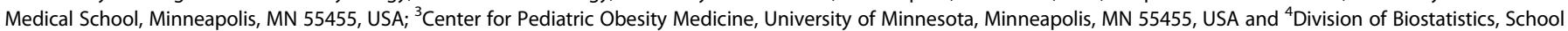
of Public Health and Clinical and Translational Science Institute, University of Minnesota, Minneapolis, MN 55455, USA Correspondence: Michelle M. Harbin (Harb0085@umn.edu)
} 
Weight Management Clinic, pediatric clinics, and from surrounding communities within the Minneapolis-St. Paul metropolitan area. Exclusion from study participation included obesity due to a genetic cause, bariatric surgery, current use of antihypertensive medications, type 1 diabetes mellitus, history of hypercholesterolemia, chronic kidney disease, Kawasaki disease, autoimmune inflammatory diseases, and congenital heart disease. The Institutional Review Board (IRB) at the University of Minnesota approved this study. All procedures followed IRB and HIPAA (Health Insurance Portability and Accountability Act) guidelines. Parents and participants provided informed consent and assent, respectively.

Determination of secondhand smoking exposure

All participants in this study were nonsmokers. Self-reported SHS exposure was dichotomized (yes/no) via a questionnaire selection asking if participants were exposed to environmental tobacco smoke at home.

\section{Anthropometric measurements}

Testing was performed at a clinical research unit at the University of Minnesota, with the participant fasted for at least $12 \mathrm{~h}$ prior to their study visit. Height and body mass were obtained three times via a wall-mounted stadiometer and an electric scale (ST Scale-Tronix, White Plains, NY, USA), and the average of the three trials was recorded. BMI was calculated as body mass in kilograms $(\mathrm{kg})$ divided by height in squared meters $\left(\mathrm{m}^{2}\right)$. Obesity status was stratified into three categories as defined by BMI percentiles: normal weight (NW; i.e., $\geq 5$ th to $<85$ th $\mathrm{BMI}$ percentile), overweight/obesity (OW/OB; i.e., $\geq 85$ th to $<95$ th BMI percentiles, $\geq 95 \%$ to $<120 \%$ of the 95 th BMI percentiles), and severe obesity (SO; i.e., $\geq 120 \%$ of the 95th BMI percentile, or absolute $\mathrm{BMI}>35 \mathrm{~kg} / \mathrm{m}^{2}$ ). Dual X-ray absorptiometry (DXA; iDXA: General Electronic Medical Systems, Madison WI, USA) was used to measure body composition; data were analyzed with enCore $^{\text {TM }}$ software (software version 16.0). Trained medical providers assessed pubertal maturation using Tanner stages (I-V).

Blood plasma lipids and inflammatory biomarkers

Blood plasma was collected via an antecubital vein for the measurement of lipids, and oxidized low-density lipoprotein (oxLDL), C-reactive protein (CRP), and adiponectin were measured at Fairview Diagnostic Laboratories, Fairview University Medical Center (Minneapolis MN, USA). All participants were free from illness and injury 2 weeks prior to study visit. All plasma samples were stored frozen at $-80^{\circ} \mathrm{C}$. OxLDL and adiponectin were analyzed at the University of Minnesota Cytokine Reference Laboratory by standard techniques via an enzyme-linked immunosorbent assay. Fairview Diagnostic Laboratories used rate nephelometry to determine CRP values.

\section{Peripheral blood pressure}

Seated brachial systolic (SBP) and diastolic blood pressure (DBP) were measured on the right arm, and after the participant rested for $10 \mathrm{~min}$, via an automated sphygmomanometer (Colin BP-8800, Colin Medical Instruments Corp., San Antonio TX, USA). Blood pressure was measured in triplicate with 3-min intervals between each measure, and the average of the last two measures was recorded. Brachial SBP and DBP percentiles were calculated as based on participant age, sex, and height. Hypertension status was determined by using current guidelines from the American Academy of Pediatrics and stratified into the following categories: normal blood pressure, elevated blood pressure, stage 1 hypertension, and stage 2 hypertension. ${ }^{21}$

Ultrasound-derived measures of arterial elasticity and stiffness Participants had vascular images measured in a quiet, temperature-controlled room $\left(22-23^{\circ} \mathrm{C}\right)$. Images of the abdominal aorta, left brachial artery, and the left carotid artery were obtained via ultrasound (Acuson, Sequoia 512, Siemens Medical Solutions
USA Inc., Mountain View, CA, USA) with a 8.0-15.0 MHz linear array probe. Following $15 \mathrm{~min}$ of supine rest, luminal systolic and diastolic diameters of the imaged artery were collected in the longitudinal plane at 20 frames per second for $10 \mathrm{~s}$, concurrently with the measurement of peripheral SBP and DBP. The left carotid artery was imaged $\sim 1 \mathrm{~cm}$ proximal from the carotid bulb, and the brachial artery was imaged 5 to $10 \mathrm{~cm}$ proximal to the antecubital fossa; the abdominal aorta was imaged distal to the renal artery and proximal to the iliac bifurcation. All artery images were digitized and stored on a personal computer for analysis using an off-line, electronic wall-tracking software program (Vascular Research Tools 6, Medical Imaging Application, LLC, lowa City, IA, USA). Mean diameter through the recording was used to calculate both distensibility and compliance. The following calculations were used for arterial distensibility, compliance, and incremental elastic modulus:

Diameter distensibility $=\left(\frac{D_{\max }-D_{\min }}{D_{\min }}\right) \times 100$,
Cross-sectional distensibility $=\left(\frac{\pi\left(0.5 \times D_{\max }\right)^{2}-\pi\left(0.5 \times D_{\min }\right)^{2}}{\pi\left(0.5 \times D_{\min }\right)^{2}}\right) \times 100$,

Diameter compliance $=\left(\frac{D_{\max }-D_{\min }}{\mathrm{PP}}\right)$,

Cross-sectional compliance $=\frac{\pi\left(0.5 \times D_{\max }\right)^{2}-\pi\left(0.5 \times D_{\min }\right)^{2}}{\mathrm{PP}}$,

Incremential elastic modulus $=\frac{3 \times\left[1+\frac{\pi\left(0.5 \times D_{\max }\right)^{2}}{\pi\left(0.5 \times D_{\min }\right)^{2}}\right]}{\mathrm{CCSC}}$.

Measurement of endothelial-dependent dilation

Endothelial-dependent vasodilation was assessed by brachial FMD. A rapidly inflating occlusion cuff (D.E. Hokanson Inc., Bellevue, WA, USA) was applied to the left forearm, $\sim 5$ to $10 \mathrm{~cm}$ distal to the antecubital fossa. Brachial artery images were obtained proximal to the antecubital fossa in the longitudinal plane, and the transducer was maintained at a constant pressure and at a fixed point over the left brachial artery with the use of a stereotactic arm. Heart rate (HR) was monitored using a 3-lead electrocardiogram, with end-diastolic images captured at the Rwave (end diastole). After obtaining vascular images of the brachial artery at rest, the occlusion cuff was inflated and maintained at a suprasystolic pressure of $200 \mathrm{mmHg}$ for $300 \mathrm{~s}$. Continuous imaging of the brachial artery started during the last $20 \mathrm{~s}$ of occlusion to $180 \mathrm{~s}$ post occlusion. Peak FMD was calculated as a 10-s average of the relative percent change from postocclusion baseline to brachial artery diameter at maximal dilation. Allometric scaling of FMD was performed in the statistical analysis, as proposed by Atkinson and Batterham. ${ }^{22}$

Measurement of aortic blood pressure and arterial stiffness Radial and carotid arterial waveforms were recorded via applanation tonometry using the SphygmoCor ${ }^{\mathrm{TM}}$ MM3 software (AtCor Medical, Sydney, Australia). The tonometer was placed over the strongest pulse point on the artery. A minimum of $11 \mathrm{~s}$ of consistent arterial waveforms were recorded after a strong and reproducible pulse was obtained. Arterial waveforms were calibrated to each participant's resting brachial blood pressure, and a validated generalized transfer function estimated the corresponding central aortic blood pressure.

Statistical analysis

IBM SPSS Statistics 23 (IBM Corp. Released 2016, IBM SPSS Statistics for Windows, Version 23, Armonk, NY: IBM Corp) was 
used for statistical analysis. Data are presented as mean \pm standard deviation (SD). Differences in vascular phenotypes between the exposed and unexposed participants were evaluated by a oneway analysis of variance, and an analysis of covariance adjusted for covariates. Associations between SHS exposure and measures of vascular phenotypes was assessed via multiple linear regression, which adjusted for Tanner stage, sex, hypertension status, race, and percent body fat (\%BF).

\section{RESULTS}

A total of 49 participants had a self-reported history of SHS exposure. Compared to unexposed participants, SHS-exposed cases were older (exposed vs. unexposed: $13.6 \pm 2.9$ vs. $12.8 \pm 2.8$ years) and had a higher BMI $\left(33.1 \pm 8.6\right.$ vs. $\left.25.8 \pm 8.4 \mathrm{~kg} / \mathrm{m}^{2}\right)$, BMl percentile $(93.7 \pm 14.0 \%$ vs. $76.3 \pm 28.7 \%)$, $\% B F(43.9 \pm 8.5 \%$ vs. $35.8 \pm 11.7 \%)$, and visceral adipose tissue ( $886.7 \pm 680.4$ vs. $448.2 \pm$ $521.3 \mathrm{~g})$ (Table 1). Brachial SBP $(119 \pm 13$ vs. $112 \pm 13 \mathrm{mmHg})$ and DBP $(62 \pm 8$ vs. $59 \pm 9 \mathrm{mmHg})$ were also higher among the exposed participants. The exposed participants had lower high-density lipoproteins $(43.5 \pm 16.1$ vs. $50.6 \pm 12.4 \mathrm{mg} / \mathrm{dL}, p<0.001)$ and higher triglycerides ( $118.2 \pm 59.7$ vs. $99.9 \pm 49.2 \mathrm{mg} / \mathrm{dL}$ ).

Unadjusted abdominal aorta diameter distensibility $(13.4 \pm 3.6 \%$ vs. $16.0 \pm 5.2 \%, p=0.009)$ and abdominal aorta cross-sectional distensibility $(28.8 \pm 8.3 \%$ vs. $35.1 \pm 12.2 \%, p=0.008)$ were significantly lower among the exposed participants; abdominal aorta incremental elastic modulus was significantly higher (1241 \pm 794 vs. $935 \pm 388 \mathrm{mmHg}, p=0.001$ ) (Table 2). Carotid-aortic SBP $(121 \pm 15$ vs. $114 \pm 14 \mathrm{mmHg}, p=0.026)$ and DBP (63 \pm 8 vs. $58 \pm 8 \mathrm{mmHg}, p=0.009$ ) were significantly higher among the SHSexposed participants; similar results were observed for radial-aortic SBP $(100 \pm 11$ vs. $95 \pm 12 \mathrm{mmHg}, p=0.030)$ and DBP (63 \pm vs. $59 \pm 9 \mathrm{mmHg}, p=0.010)$. With regard to systemic inflammation, oxLDL was significantly higher among the exposed participants $(p=0.044)$, and adiponectin was significantly lower $(p=0.013)$. SHS exposure was not associated with measures of arterial elasticity, including distensibility, compliance, and incremental elastic modulus, in the brachial and carotid artery. Carotid-radial pulse wave velocity $(p=0.455)$, as well as FMD $(p=0.239)$, was not significantly different between the unexposed and exposed participants.

Multiple linear regression analysis report that, following adjustment for Tanner stage, sex, race, and hypertension status, SHS exposure was associated with increased abdominal aorta incremental elastic modulus $(\beta=273.7, p=0.004)$, decreased abdominal aorta distensibility $(\beta=-2.2, \quad p=0.025)$, and decreased abdominal aorta cross-sectional distensibility $(\beta=-5.4$, $p=0.023$ ) (Table 3). With the additional adjustment of \%BF, significance persisted for abdominal aorta incremental elastic modulus ( $1184 \pm 474$ vs. $954 \pm 469 \mathrm{mmHg}, p=0.017)$, diameter distensibility $(13.9 \pm 5.1 \%$ vs. $15.9 \pm 5.1 \%, p=0.047)$, and crosssectional distensibility $(29.9 \pm 12.0 \%$ vs. $34.9 \pm 12.0 \%, p=0.040)$ (Fig. 1). Differences in carotid-aortic SBP $(p=0.399)$ and DBP $(p=$ $0.058)$, as well as radial-aortic SBP $(p=0.617)$ and DBP $(p=0.146)$, were minimized with adjusted for Tanner stage, sex, race, hypertension status, and \%BF; similar results were reported for oxLDL $(p=0.440)$ and adiponectin $(p=0.625)$.

\section{DISCUSSION}

Exposure to SHS among children and adolescents was associated with increased arterial stiffness in the abdominal aorta, while the majority of vascular measures in the carotid and brachial arteries were unaffected. These data suggest that exposure to SHS may predispose children and adolescents to subclinical CVD risk, which may first appear in the abdominal aorta, an artery that previous research has shown to be affected by atherosclerosis earlier than other vascular beds. ${ }^{23}$ In addition, both carotid-aortic SBP and
Table 1. Cohort demographics and anthropometrics

\begin{tabular}{|c|c|c|}
\hline & Exposed $(n=49)$ & Unexposed $(n=249)$ \\
\hline Age (years) & $13.6 \pm 2.9$ & $12.8 \pm 2.8$ \\
\hline Male sex ( $n[\%])$ & $19(38.8)$ & $124(49.8)$ \\
\hline \multicolumn{3}{|l|}{ Race $(n[\%])$} \\
\hline White & $36(73.5)$ & $221(88.7)$ \\
\hline Black & $11(22.4)$ & $21(8.4)$ \\
\hline Other & $2(4.1)$ & $7(2.8)$ \\
\hline \multicolumn{3}{|l|}{ Tanner stage $(n[\%])$} \\
\hline 1 & $5(10.2)$ & $70(28.1)$ \\
\hline$\|$ & $10(20.4)$ & $48(19.2)$ \\
\hline III & $12(24.5)$ & $35(14.1)$ \\
\hline IV & $14(28.6)$ & $50(20.1)$ \\
\hline V & $8(16.3)$ & $45(18.1)$ \\
\hline Body mass (kg) & $87.9 \pm 31.8$ & $65.7 \pm 28.7$ \\
\hline Height (cm) & $161.1 \pm 12.2$ & $156.8 \pm 14.4$ \\
\hline BMI $\left(\mathrm{kg} / \mathrm{m}^{2}\right)$ & $33.1 \pm 8.6$ & $25.8 \pm 8.4$ \\
\hline BMI percentile (\%) & $93.7 \pm 14.0$ & $76.3 \pm 28.7$ \\
\hline \multicolumn{3}{|l|}{ Obesity status ( $n[\%]$ ) } \\
\hline Normal weight & $6(12.2)$ & $104(41.8)$ \\
\hline Overweight/obesity & $11(22.4)$ & $72(28.9)$ \\
\hline Severe obesity & $33(65.3)$ & $73(29.3)$ \\
\hline Hip circumference $(\mathrm{cm})$ & $106.6 \pm 17.7$ & $93.3 \pm 18.4$ \\
\hline Waist circumference $(\mathrm{cm})$ & $95.4 \pm 20.1$ & $78.6 \pm 18.1$ \\
\hline Lean muscle mass (kg) & $46.3 \pm 13.9$ & $38.6 \pm 13.4$ \\
\hline Fat mass $(\mathrm{kg})$ & $38.9 \pm 19.1$ & $24.6 \pm 17.0$ \\
\hline Percent body fat (\%) & $43.9 \pm 8.5$ & $35.8 \pm 11.7$ \\
\hline Visceral adipose tissue (g) & $886.7 \pm 680.4$ & $448.2 \pm 521.3$ \\
\hline $\begin{array}{l}\text { Systolic blood } \\
\text { pressure }(\mathrm{mmHg})\end{array}$ & $119 \pm 13$ & $112 \pm 12$ \\
\hline $\begin{array}{l}\text { Diastolic blood } \\
\text { pressure }(\mathrm{mmHg})\end{array}$ & $62 \pm 8$ & $59 \pm 8$ \\
\hline \multicolumn{3}{|l|}{ Hypertension status ( $n$ [\%]) } \\
\hline Normal blood pressure & $28(57.1)$ & $190(76.3)$ \\
\hline Elevated blood pressure & $9(18.4)$ & $31(12.4)$ \\
\hline Stage I hypertension & $6(12.2)$ & $13(5.2)$ \\
\hline Stage II hypertension & $6(12.2)$ & $15(6.0)$ \\
\hline Heart rate (b.p.m.) & $75 \pm 10$ & $75 \pm 11$ \\
\hline Total cholesterol (mg/dL) & $159.6 \pm 28.1$ & $159.8 \pm 27.7$ \\
\hline $\begin{array}{l}\text { Low-density lipoprotein } \\
\text { (mg/dL) }\end{array}$ & $92.4 \pm 25.0$ & $89.3 \pm 25.0$ \\
\hline $\begin{array}{l}\text { Very low-density lipoprotein } \\
\text { (mg/dL) }\end{array}$ & $24.3 \pm 12.1$ & $21.1 \pm 10.4$ \\
\hline $\begin{array}{l}\text { High-density lipoprotein } \\
\text { (mg/dL) }\end{array}$ & $43.5 \pm 16.1$ & $50.6 \pm 13.4$ \\
\hline Triglycerides (mg/dL) & $118.2 \pm 59.7$ & $99.9 \pm 49.2$ \\
\hline
\end{tabular}

$B M I$, body mass index

Continuous variables are presented as mean \pm standard deviation (SD) Categorical variables (e.g., race, Tanner stage, obesity status, hypertension status) are presented as count (\% within column)

Obesity status is presented as normal weight (e.g., $\geq 5$ th to $<85$ th BMI percentile), overweight/obesity (e.g., $\geq 85$ th to $<95$ th BMI percentile, $\geq 100 \%$ to $<120 \%$ of the 95 th BMI percentile), and severe obesity (e.g., $\geq 120 \%$ of the 95th BMI percentile)

Hypertension status was determined by using the current recommendations from the American Academy of Pediatrics and stratified into the following categories: normal blood pressure, elevated blood pressure, stage I hypertension, and stage II hypertension 
Table 2. Comparisons in vascular structure and function between non-exposed and exposed youth to secondhand smoke

\begin{tabular}{|c|c|c|c|c|c|}
\hline & $n$ & Exposed & $n$ & Unexposed & $P$ value \\
\hline Abdominal aorta (mm) & 29 & $10.2 \pm 1.9$ & 191 & $9.6 \pm 2.2$ & 0.153 \\
\hline Carotid artery $(\mathrm{mm})$ & 48 & $6.5 \pm 0.6$ & 238 & $6.3 \pm 0.7$ & 0.150 \\
\hline \multicolumn{6}{|l|}{ Incremental elastic modulus } \\
\hline Carotid artery $(\mathrm{mmHg})$ & 48 & $1184 \pm 388$ & 238 & $1008 \pm 332$ & 0.078 \\
\hline \multicolumn{6}{|l|}{ Diameter distensibility } \\
\hline Abdominal aorta (\%) & 29 & $13.4 \pm 3.6$ & 191 & $16.0 \pm 5.2$ & $0.009^{*}$ \\
\hline Brachial artery (\%) & 40 & $3.5 \pm 1.2$ & 151 & $3.8 \pm 2.0$ & 0.432 \\
\hline Carotid artery (\%) & 48 & $13.1 \pm 3.1$ & 238 & $14.1 \pm 7.1$ & 0.336 \\
\hline \multicolumn{6}{|l|}{ Diameter compliance } \\
\hline Abdominal aorta $(\mathrm{mm} / \mathrm{mmHg})$ & 29 & $0.03 \pm 0.01$ & 191 & $0.03 \pm 0.01$ & 0.320 \\
\hline Brachial artery $(\mathrm{mm} / \mathrm{mmHg})$ & 40 & $0.002 \pm 0.002$ & 151 & $0.003 \pm 0.003$ & 0.890 \\
\hline Carotid artery $(\mathrm{mm} / \mathrm{mmHg})$ & 48 & $0.01 \pm 0.003$ & 238 & $0.02 \pm 0.008$ & 0.258 \\
\hline \multicolumn{6}{|l|}{ Cross-sectional compliance } \\
\hline Abdominal aorta $\left(\mathrm{mm}^{2} / \mathrm{mmHg}\right)$ & 29 & $0.4 \pm 0.2$ & 191 & $0.5 \pm 0.2$ & 0.690 \\
\hline Brachial artery $\left(\mathrm{mm}^{2} / \mathrm{mmHg}\right)$ & 40 & $0.01 \pm 0.01$ & 151 & $0.02 \pm 0.06$ & 0.654 \\
\hline Carotid artery $\left(\mathrm{mm}^{2} / \mathrm{mmHg}\right)$ & 48 & $0.2 \pm 0.05$ & 238 & $0.02 \pm 0.04$ & 0.583 \\
\hline \multicolumn{6}{|l|}{ Aortic blood pressure } \\
\hline Carotid-aortic SBP (mmHg) & 31 & $121 \pm 15$ & 133 & $114 \pm 14$ & $0.026^{*}$ \\
\hline $\mathrm{FMD}_{\mathrm{AUC}}(\% / \mathrm{s})$ & 46 & $757 \pm 524$ & 213 & $671 \pm 497$ & 0.296 \\
\hline C-reactive protein $(\mathrm{mg} / \mathrm{L})$ & 48 & $7.2 \pm 9.4$ & 217 & $5.5 \pm 9.0$ & 0.255 \\
\hline Oxidized LDL (U/L) & 48 & $62.5 \pm 39.6$ & 238 & $51.9 \pm 31.3$ & $0.044^{*}$ \\
\hline Adiponectin $(\mu \mathrm{g} / \mathrm{mL})$ & 48 & $2.9 \pm 2.0$ & 239 & $4.0 \pm 2.9$ & $0.013^{*}$ \\
\hline
\end{tabular}

DBP, as well as radial-aortic SBP and DBP, were higher among the exposed cases, although differences were minimized after adjustment for covariates.

Mechanisms of vascular disruption from SHS are attributed to toxins that can vary in composition and potency, among which includes nicotine, acrolein, crotonaldehyde, cadmium, lead, and particular matter. ${ }^{12}$ While toxins in SHS are more diluted as compared to active smoking, it may be just as harmful due to longer exposure to residual toxins that contain pro-inflammatory, pro-thrombotic, and oxidative properties. ${ }^{24-26}$ Past research has observed increased arterial stiffness, ${ }^{6-8,27,28}$ increased carotid intima-media thickness, ${ }^{29}$ decreased endothelial function, ${ }^{3,4}$ and increased coronary artery calcification ${ }^{30,31}$ among nonsmoking adults with SHS exposure. The Young Finns study reported that parental smoking among children and adolescents between 3 and 18 years of age was associated with reduced brachial FMD later in adulthood (28-45 years old), suggesting that environmental exposure to tobacco smoke in childhood may induce irreversible impairment in vascular function. ${ }^{32}$ In this present study, we report no differences in brachial FMD regardless of SHS exposure. These discrepancies in endothelial function between our study with previous reports may be attributable to differences in the age of the cohorts. While the Young Finns study evaluated the effect of SHS exposure among adults that were exposed during young 
Table 3. Adjusted associations of secondhand smoking with measures of aortic blood pressure and vascular structure and elasticity

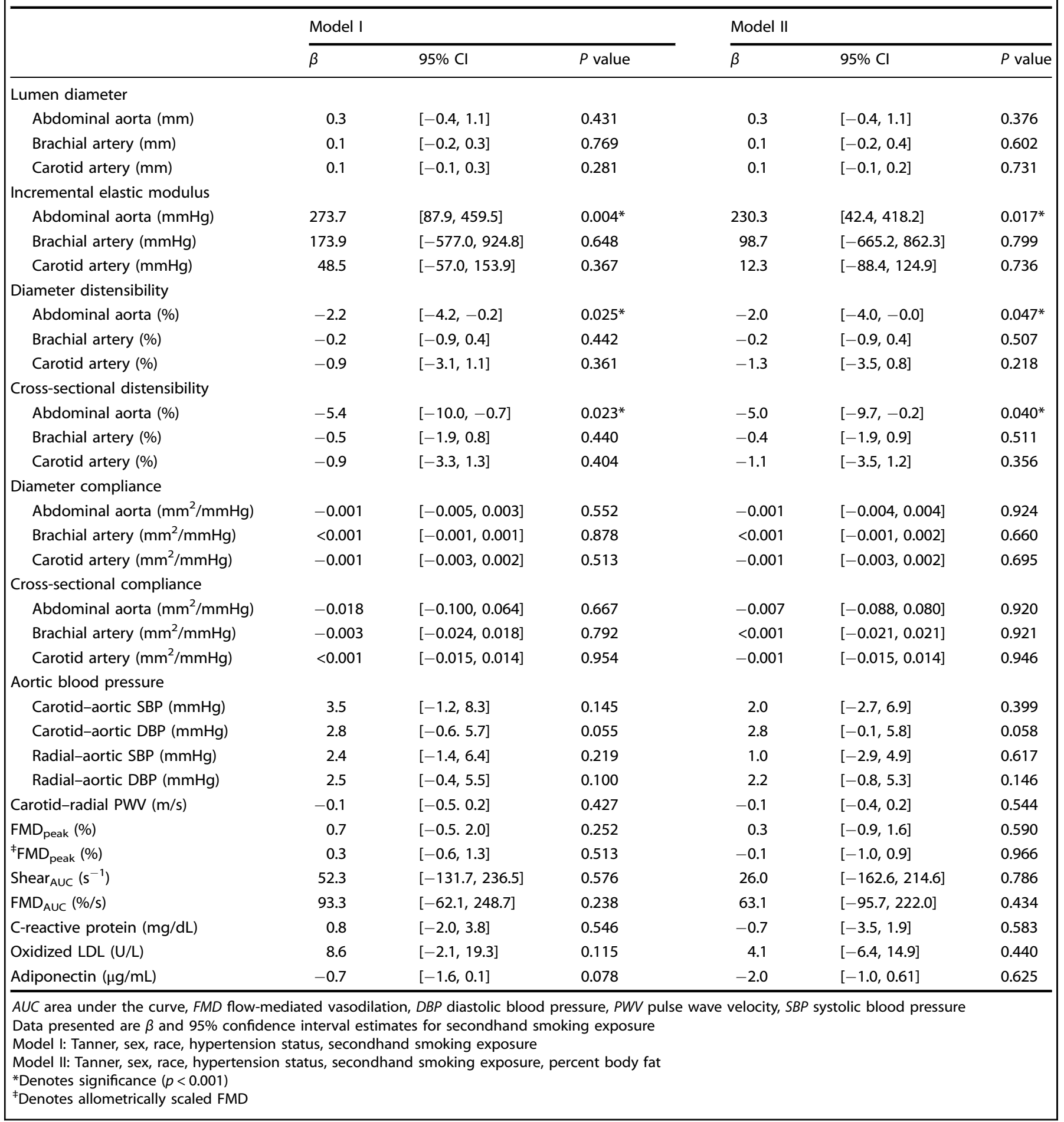

childhood and adolescence, ${ }^{32}$ our study measured brachial FMD among children and adolescents, which is suggestive that impairments in endothelial function may not become apparent until adulthood. Additionally, the Young Finns study observed that children and adolescents with parents who smoked had increased risk of developing carotid artery atherosclerotic lesions in adulthood (average age at follow-up: 36.6 years old). ${ }^{18}$ There is also evidence that SHS is detrimental during young childhood, including fetal development. ${ }^{33-35}$ A meta-analysis reported that maternal SHS exposure was associated with low birth weight, smaller head circumferences, and congenital anomalies. ${ }^{36}$ The current understanding from the literature is that the risk from SHS may be occult during childhood and adolescence, but can predispose such individuals to substantial increased risk of CVD later in adulthood.

The effect of SHS on arterial stiffness has predominately been restricted to analysis of arterial wave reflection to the aorta among adults. ${ }^{27}$ Previous reports observed that passive smoking results in increased aortic blood pressure in adults; the increase in augmentation index was only observed among males. ${ }^{7}$ Using 

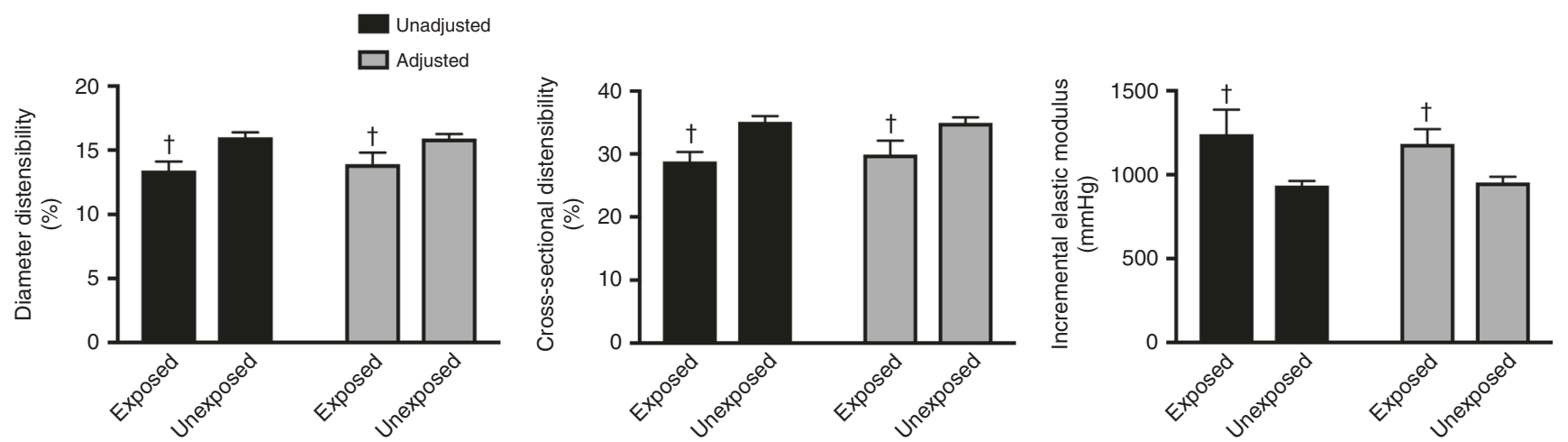

Fig. 1 Mean \pm standard error (SE). Analysis of covariance (ANCOVA) adjusted for Tanner stage, sex, hypertension status, race, and percent body fat. ${ }^{\dagger}$ Denotes significance $(p<0.05)$

invasive catheterization, both active smoking and passive SHS resulted in decreased aortic mean distensibility among adults. ${ }^{8}$ With regard to children and adolescents, one study reported that higher cotinine concentrations were associated with increased ultrasound-derived measures of aortic Young's elastic modulus, as well as decreased carotid distensibility. ${ }^{19}$ This current study adds to the current literature by providing ultrasound-derived measures of arterial compliance, distensibility, and incremental elastic modulus within the brachial, carotid, and abdominal aorta among a young cohort with varying ages and BMI levels.

We report increased abdominal aorta stiffness among children and adolescents with a self-reported history of SHS exposure. Adjusted measures of abdominal aorta distensibility were significantly lower among the exposed cases. Measures of elasticity and arterial stiffness within the carotid and brachial artery were not significantly affected with SHS exposure. This finding is in agreement with preliminary reports from Kawasaki et al. ${ }^{37}$ that arterial stiffness increased with age within the abdominal aorta, but not within brachial and femoral arteries. Lanne et al. ${ }^{23}$ similarly reported regional differences in changes arterial compliance with age; in particular, the abdominal aorta exhibited greater increases in arterial stiffness with age as compared to the carotid artery among Caucasian males. While the current study evaluated the effect of SHS, and not age, on arterial elasticity and stiffness, we similarly report that degenerative changes indicative of increased arteriosclerosis risk occurs first within the deep elastic arteries (i.e., abdominal aorta) prior to the peripheral arteries.

Despite the fact that SHS exposure has declined within the general population during the past 30 years, an estimated 24 million nonsmoking children and adolescents in the United States are currently exposed. ${ }^{38}$ Furthermore, it is well recognized that parental smoking is a major contributor of SHS exposure. Racial disparities also persist; the decline in SHS exposure between the years 1999 to 2000 and 2011 to 2012 was substantially less among non-Hispanic Blacks as compared to both non-Hispanic Whites and Hispanic children and adolescents. ${ }^{38}$ Strengths of this study include a large sample size, the use of DXA for measures of body composition, and the use of ultrasound imaging for measures arterial elasticity and stiffness within the abdominal aorta, carotid, and brachial artery. A limitation of this analysis was that SHS exposure was self-reported, and the cross-sectional nature prevents us from suggesting causality between SHS and abdominal aorta stiffness. A third limitation was that exposure was dichotomized into exposed and unexposed categories, precluding us from evaluating a dose-response relationship between the duration and frequency of SHS exposure on vascular structure and function.

\section{CONCLUSION}

In summary, children and adolescents with self-reported exposure to SHS had greater stiffness in the abdominal aorta, but not other vascular beds, than unexposed children and adolescents. Importantly, this study provides additional evidence supporting the association of smoking with CVD risk during childhood, which may be an ideal timing for active and passive smoking exposure prevention programs to have optimal impact for reducing CVD risk related to smoke exposure.

\section{ACKNOWLEDGEMENTS}

Funding for this project was provided by the National Heart, Lung, and Blood Institute (R01HL110957, awarded to A.S.K.).

\section{AUTHOR CONTRIBUTIONS}

No payment was received to produce this manuscript. M.M.H. drafted the manuscript. M.M.H. and J.R.R. developed the aims and hypothesis. A.S.K. and J.R.R. provided the funding, and contributed to the conception, design, and analysis and interpretation of the data. N.G.E. was involved with the acquisition of the data. K.D.R., A.S.K., D.R.D., N.G.E., and J.R.R. provided subject matter expertise and a critical review of the manuscript. All authors have seen this manuscript, and give approval of this version to be published.

\section{ADDITIONAL INFORMATION}

Competing interests: Dr. Kelly serves as a consultant for Novo Nordisk, WW, and Vivus Pharmaceuticals, but does not receive personal or professional income for these activities; he also receives research support (drug/placebo) from Astra Zeneca Pharmaceuticals for an NIDDK-funded clinical trial. Dr. Dengel serves as a paid consultant for Hologic Inc. Dr. Ryder receives research support (drug/placebo) from Boheringer Ingelheim for a clinical trial. The other authors declare no competing interests.

Publisher's note Springer Nature remains neutral with regard to jurisdictional claims in published maps and institutional affiliations.

\section{REFERENCES}

1. Barnoya, J. \& Glantz, S. A. Cardiovascular effects of secondhand smoke: nearly as large as smoking. Circulation 111, 2684-2698 (2005).

2. Davis, J. W., Shelton, L., Watanabe, I. S. \& Arnold, J. Passive smoking affects endothelium and platelets. Arch. Intern. Med. 149, 386-389 (1989).

3. Otsuka, R. et al. Acute effects of passive smoking on the coronary circulation in healthy young adults. JAMA 286, 436-441 (2001).

4. Celermajer, D. S. et al. Passive smoking and impaired endothelium-dependent arterial dilatation in healthy young adults. N. Engl. J. Med. 334, 150-154 (1996).

5. Sumida, H. et al. Does passive smoking impair endothelium-dependent coronary artery dilation in women? J. Am. Coll. Cardiol. 31, 811-815 (1998).

6. Mack, W. J., Islam, T., Lee, Z., Selzer, R. H. \& Hodis, H. N. Environmental tobacco smoke and carotid arterial stiffness. Prev. Med. 37, 148-154 (2003). 
7. Mahmud, A. \& Feely, J. Effects of passive smoking on blood pressure and aortic pressure waveform in healthy young adults-influence of gender. Br. J. Clin. Pharm. 57, 37-43 (2004).

8. Stefanadis, C. et al. Unfavorable effects of passive smoking on aortic function in men. Ann. Intern. Med. 128, 426-434 (1998).

9. Panagiotakos, D. B. et al. Effect of exposure to secondhand smoke on markers of inflammation: the ATTICA study. Am. J. Med. 116, 145-150 (2004).

10. Moritsugu, K. P. The 2006 Report of the Surgeon General: the health consequences of involuntary exposure to tobacco smoke. Am. J. Prev. Med. 32, 542-543 (2007).

11. Tsai, J. et al. Exposure to secondhand smoke among nonsmokers-United States, 1988-2014. Morb. Mortal. Wkly Rep. 67, 1342-1346 (2018).

12. Raghuveer, G. et al. Cardiovascular consequences of childhood secondhand tobacco smoke exposure: prevailing evidence, burden, and racial and socioeconomic disparities: a scientific statement from the american heart association. Circulation 134, e336-e359 (2016).

13. Bandiera, F. C., Richardson, A. K., Lee, D. J., He, J. P. \& Merikangas, K. R. Secondhand smoke exposure and mental health among children and adolescents. Arch. Pediatr. Adolesc. Med. 165, 332-338 (2011).

14. Chen, R., Clifford, A., Lang, L. \& Anstey, K. J. Is exposure to secondhand smoke associated with cognitive parameters of children and adolescents?-a systematic literature review. Ann. Epidemiol. 23, 652-661 (2013).

15. Kabir, Z., Connolly, G. N. \& Alpert, H. R. Secondhand smoke exposure and neurobehavioral disorders among children in the United States. Pediatrics 128, 263-270 (2011).

16. Weitzman, M. et al. Tobacco smoke exposure is associated with the metabolic syndrome in adolescents. Circulation 112, 862-869 (2005).

17. Mannino, D. M., Moorman, J. E., Kingsley, B., Rose, D. \& Repace, J. Health effects related to environmental tobacco smoke exposure in children in the United States: data from the Third National Health and Nutrition Examination Survey. Arch. Pediatr. Adolesc. Med. 155, 36-41 (2001).

18. West, H. W. et al. Exposure to parental smoking in childhood is associated with increased risk of carotid atherosclerotic plaque in adulthood: the Cardiovascular Risk in Young Finns Study. Circulation 131, 1239-1246 (2015).

19. Kallio, K. et al. Arterial intima-media thickness, endothelial function, and apolipoproteins in adolescents frequently exposed to tobacco smoke. Circ. Cardiovasc. Qual. Outcomes 3, 196-203 (2010).

20. Simonetti, G. D. et al. Determinants of blood pressure in preschool children: the role of parental smoking. Circulation 123, 292-298 (2011).

21. Flynn, J. T. et al. Clinical practice guideline for screening and management of high blood pressure in children and adolescents. Pediatrics 140, 1-72 (2017).

22. Atkinson, G. \& Batterham, A. M. Allometric scaling of diameter change in the original flow-mediated dilation protocol. Atherosclerosis 226, 425-427 (2013).

23. Lanne, T., Hansen, F., Mangell, P. \& Sonesson, B. Differences in mechanical properties of the common carotid artery and abdominal aorta in healthy males. J. Vasc. Surg. 20, 218-225 (1994).
24. Glantz, S. A. \& Parmley, W. W. Passive smoking and heart disease. Mechanisms risk. JAMA 273, 1047-1053 (1995).

25. Glantz, S. A. \& Parmley, W. W. Passive smoking and heart disease. Epidemiol. Physiol. Biochem. Circ. 83, 1-12 (1991).

26. Rubenstein, D., Jesty, J. \& Bluestein, D. Differences between mainstream and sidestream cigarette smoke extracts and nicotine in the activation of platelets under static and flow conditions. Circulation 109, 78-83 (2004).

27. Argacha, J. F. et al. Acute effects of passive smoking on peripheral vascular function. Hypertension 51, 1506-1511 (2008).

28. Gac, P., Poreba, M., Mazur, G. \& Poreba, R. The aortic mechanical properties in patients with the essential hypertension environmentally exposed to cigaret smoke. Inhal. Toxicol. 27, 717-723 (2015).

29. Chen, W. et al. Secondhand smoke exposure is associated with increased carotid artery intima-media thickness: the Bogalusa Heart Study. Atherosclerosis 240, 374-379 (2015).

30. Peinemann, F. et al. Secondhand smoke exposure and coronary artery calcification among nonsmoking participants of a population-based cohort. Environ. Health Perspect. 119, 1556-1561 (2011).

31. Yankelevitz, D. F. et al. Second-hand tobacco smoke in never smokers is a significant risk factor for coronary artery calcification. JACC Cardiovasc. Imag. 6, 651-657 (2013)

32. Juonala, M. et al. Parental smoking in childhood and brachial artery flowmediated dilatation in young adults: the Cardiovascular Risk in Young Finns study and the Childhood Determinants of Adult Health study. Arterioscler. Thromb. Vasc. Biol. 32, 1024-1031 (2012).

33. Wang, L., Mamudu, H. M., Alamian, A., Anderson, J. L. \& Brooks, B. Independent and joint effects of prenatal maternal smoking and maternal exposure to secondhand smoke on the development of adolescent obesity: a longitudinal study. J. Paediatr. Child Health 50, 908-915 (2014).

34. Wahabi, H. A., Mandil, A. A., Alzeidan, R. A., Bahnassy, A. A. \& Fayed, A. A. The independent effects of second hand smoke exposure and maternal body mass index on the anthropometric measurements of the newborn. BMC Public Health 13, 1058 (2013)

35. Wahabi, H. A. et al. Effects of secondhand smoke on the birth weight of term infants and the demographic profile of Saudi exposed women. BMC Public Health 13, 341 (2013).

36. Salmasi, G., Grady, R., Jones, J. \& McDonald, S. D. Environmental tobacco smoke exposure and perinatal outcomes: a systematic review and meta-analyses. Acta Obstet. Gynecol. Scand. 89, 423-441 (2010).

37. Kawasaki, T., Sasayama, S., Yagi, S., Asakawa, T. \& Hirai, T. Non-invasive assessment of the age related changes in stiffness of major branches of the human arteries. Cardiovasc. Res. 21, 678-687 (1987).

38. Homa, D. M. et al. Vital signs: disparities in nonsmokers' exposure to secondhand smoke-United States, 1999-2012. Morb. Mortal. Wkly Rep. 64, 103-108 (2015). 